\title{
Propuesta de Gestión de la Cadena de Suministro Centralizada. Comparación con la Gestión de la Cadena de Suministro Totalmente Integrada
}

\author{
Oscar Rubiano Ovalle*
}

* Ph.D. Profesor de la Escuela de Ingeniería Industrial y Estadística - Facultad de Ingeniería Universidad del Valle Santiago de Cali, Colombia.

E-mail:oscaruba@pino.univalle.edu.co

Fecha de recepción: Octubre 27 de 2003

Fecha de aprobación: Abril 30 de 2004

En este artículo se presenta una alternativa para mejorar el rendimiento en una cadena de suministro (CS), al introducir la cadena de suministro centralizada, la cual implica una gestión global de la cadena mediante un enfoque basado en el sistema de gestión COMWIP. Para esto se construyó un modelo de simulación para explorar y evaluar las ventajas de esta estrategia, comparándola con la cadena del suministro totalmente integrada (CS TI). El análisis se realiza para diferentes niveles de variabilidad de la demanda y para posibles restricciones de flujo de material a lo largo de la cadena. Esta 
investigación muestra que esta estrategia de centralización puede ofrecer las ventajas y rendimientos mejorados en la métrica global cuando ambas estrategias ofrecen niveles de servicio similares. Las ventajas principales son: menor impacto de variabilidad de la demanda en la política de ordenar y menores niveles de inventario medio de productos terminados (IPT) y trabajo en proceso (IPP).

Palabras claves: COMWIP, Gestión de la cadena de suministro (GCS), Control centralizado, Simulación, Dinámica de Sistemas.

\section{ABSTRACT}

An alternative is presented in this paper to improve the performance in a supply chain (CS), when introducing the so-called centralized supply chain. This implies a global management based on the COMWIP-proceeding system. A simulation model was designed to explore and evaluate the comparative advantages of this strategy in front the performance of the fully integrated supply chain (CS TI). The analysis is carried out for different levels of variability in demand and with possible materials-flow restrictions along the chain. This research can show the advantages and improved performance that this centralization system actually has in the global metrics whenever both strategies are offering similar levels of service. Its main advantages are: 1. A lower impact from the variability in demand on the ordering policies, and 2. Lower levels of average finished-goods and work in process (FGI+WIP) levels.

Key words: COMWIP, Supply Chain Management, Centralized Control, Simulation, System Dynamics

\section{INTRODUCCIÓN}

La cadena de suministro COMWIP es un enfoque mediante el cual se intenta mejorar el rendimiento de Cadena de Suministro, a través de una extensión del sistema de control de producción cerrado COMWIP-COMstant Work In Process (Spearman et al. 1990).
COMWIP es una técnica de producción "long-pull", que constituye una generalización del sistema del kanban. En los sistemas COMWIP las tarjetas se asignan a la línea de producción global (Spearman et al. 1990). Al comenzar la producción, todas las tarjetas disponibles se localizan al comienzo de la línea (en un casillero de tarjetas). Al llegar órdenes, si hay suficientes tarjetas disponibles en el sistema, las tarjetas necesarias se adhieren a éstas y juntas se desplazan a través de la línea de producción. Cuando la orden se procesa completamente en la línea, y sale del último centro de trabajo, la tarjeta es removida y enviada al comienzo de la línea. Minguna orden puede entrar en la línea sin habérsele asignado tarjetas, es decir, si llegan órdenes y no hay tarjeta libre, las órdenes se acumulan como ordenes pendientes, de donde se descargarán a medida que se liberan tarjetas por concepto de ordenes terminadas. Se establecen búferes intermedios entre dos estaciones consecutivas, gestionados por una disciplina PEPS (primero en llegar, primero en ser servido).

El mecanismo de control de flujo detallado del COMWIP es discutido extensamente por Hopp y Spearman (1996). La figura 1 muestra un esquema operativo de los sistemas pull y COMWIP, en donde la variable li representa la entrada a la compañía representada por el nodo i, de la misma manera, $\mathrm{Pi}$ es el inventario en proceso, Oi es la tasa de producción, Yi es el inventario de producto terminado y $\mathrm{Si}$ es la entrega al nodo siguiente.

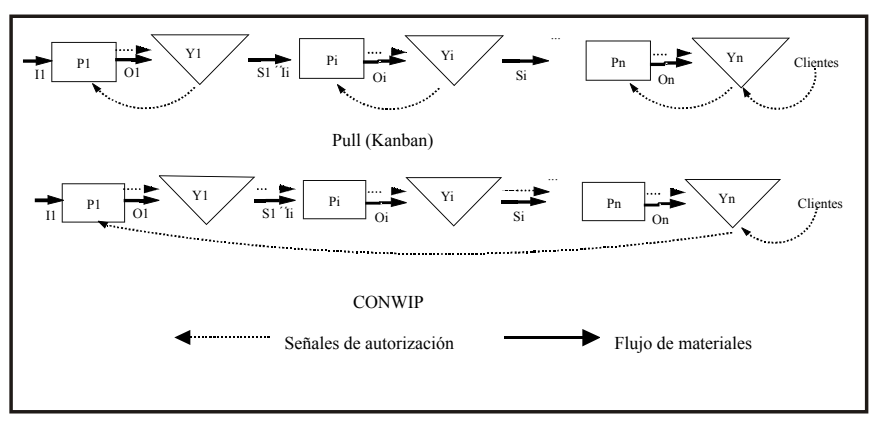

Figura 1. Esquemas de los sistemas Pull y COMWIP 
La cadena de suministro COMWIP se define como un sistema de producción-distribución, en el cual la línea de producción de cada compañía o nodo es una etapa que hace parte de la línea de suministro global. A medida que llegan los pedidos de los clientes finales, se lanzan órdenes y partes, teniendo en cuenta las restricciones de capacidad y disponibilidad de tarjetas de producción. Ordenes, tarjetas y partes, fluyen desde la primera hasta la última compañía. El casillero de tarjetas mencionado en la descripción del sistema COMWIP, se extiende a un centro virtual de control, que gobierna la cadena de suministro y administra los flujos de materiales y los inventarios a lo largo de la cadena.

Cuando las órdenes llegan a los detallistas, se lanzan las órdenes de producción y los materiales requeridos se liberan al primer miembro aguas arriba (primer centro de trabajo proveedor, en este caso, el primer proceso que transforma la materia prima) de la cadena de valor, teniendo en cuenta sus restricciones de capacidad de producción (ver figura 2). Hay un único y centralizado control de órdenes pendientes para toda la CS. Por lo tanto, el control de información centralizado mediante herramientas de comunicación, es crítico en este contexto.

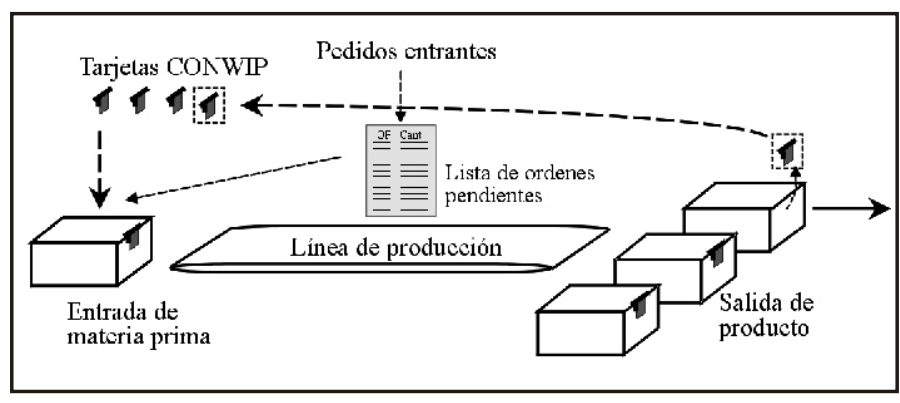

Figura 2. El uso de tarjetas con la línea COMWIP

En la siguiente sección, se revisan primero las principales contribuciones relativas a los aspectos de integración de la cadena de suministro. En la sección 3 se presenta una revisión del sistema de COMWIP y luego se discute la CS COMWIP. En la sección 4, se caracteriza un modelo de una CS COMWIP y se compara con un modelo de una CS TI. En la sección 5, se discuten los resultados de correr el modelo de simulación de la CS COMWIP, para validar el comportamiento de las principales variables. En la sección 6 , se seleccionan ciertas métricas, así mismo, se obtienen y discuten sus resultados para evaluar y comparar la CS COMWIP con la CS TI. Finalmente se presentan las conclusiones en la sección 7.

\section{ASPECTOS RELEVANTES CON RESPECTO A LA INTEGRACIÓN DE LA CADENA DE SUMINISTRO}

Rubiano (2003), presenta una recopilación suficiente sobre la importancia de la gestión de información en la integración y la mejora de los rendimientos en las cadenas de suministro. El autor presenta también un modelo para medir las mejoras en la gestión de la cadena de suministro, como una consecuencia de un mayor flujo de información compartido y evalúa el impacto de las nuevas tecnologías de la comunicación sobre el rendimiento operativo de una cadena de suministro genérica. El autor muestra cómo al utilizar las herramientas de integración, las compañías de la cadena ganan visibilidad de la información de la demanda y los inventarios a lo largo de la cadena misma, mejorando la efectividad global de la CS (throughput), así como la eficacia (nivel de servicio), neutralizando significativamente al mismo tiempo, el efecto bullwhip.

La particularidad de este modelo radica en que todas las compañías de la cadena tienen acceso a información común, pero todas toman sus decisiones en forma independiente, es decir en forma aquí llamada "descentralizada". Esto significa que no existe un ente coordinador de las operaciones a lo largo de la cadena.

\section{3. ¿POR QUÉ UNA ESTRATEGIA DE CENTRALIZACIÓN CONWIP?}

Varios autores destacan las bondades del sistema COMWIP como un arma competitiva. Se 
citan aquí algunos estudios en los cuales se muestra al COMWIP como un sistema de características superiores a las de otros sistemas como los sistemas push y los sistemas pull puros. Se destacan las siguientes:

च Es más sencillo en el sentido que se requiere un único control de tarjetas, en lugar de un control para cada estación de trabajo (Hopp y Spearman 1996).

v Puede gestionar y suministrar una mezcla de productos variable, debido al uso de tarjetas específicas en las líneas y al manejo de un registro de órdenes pendientes de lanzar a la producción (Hopp y Spearman 1996).

$\square \quad$ Introduce menos tensión al operario debido a un ambiente productivo más flexible (Hopp y Spearman 1996).

$\square$ En una línea de flujo que produce un solo tipo de parte, Spearman y Zazanis (1992) mostraron que el sistema COMWIP produce un "throughput" (ventas) medio más alto que el Kanban. En el mismo escenario, Muckstadt y Tayur (1995a, 1995b) mostraron que el COMWIP produce un throughput menos variable y un inventario máximo más bajo que el Kanban.

■ Según un estudio de simulación llevado a cabo por Roderick et al. (1990), el sistema COMWIP es superior a otros sistemas de control de producción en lo relativo a las fechas de entrega prometidas y a los tiempos de ciclo.

En comparación con la CS TI, podrían capitalizarse las ventajas siguientes al introducir elementos COMWIP (de acuerdo con la revisión realizada de la literatura) a la GCS. Las compañías intermedias de una cadena de suministro:

च Mo necesitarían establecer políticas de lanzamiento de órdenes, lo cual evitaría la amplificación del pulso de la demanda a lo largo de la cadena, sólo un ente central autorizaría la liberación de trabajo con base en el estado del sistema, definido por el número total de tarjetas "adjuntas" a las órdenes.
■ Tomarían decisiones operativas más simples y realizarían un control más fácil del flujo de materiales y el trabajo en proceso (bajo las condiciones normales, en una cadena del suministro centralizada, las empresas simplemente procesarían y transferirían la totalidad de las partes recibidas, haciendo que las partes justamente fluyan a lo largo de la cadena mediante un efecto push).

\section{MODELADO DE CS CONWIP Y COMPARACIÓN CON EL MODELO DE LA CS TOTALMENTE INTEGRADA}

Para el presente estudio, se realizó un modelo de simulación con Dinámica de sistemas, en el cual, para el flujo físico en una compañía se consideran dos variables de estado, los niveles de trabajo en proceso y los niveles de inventario de partes terminadas enfrente de la próxima compañía aguas abajo (cliente). En el flujo de información, se consideran dos únicas variables de estado: los pedidos no satisfechos a tiempo y los pedidos no lanzados a producción a tiempo.

A continuación se describe la notación de las principales variables del modelo:

\section{$\square$ Variables del flujo de material:}
$P_{t}^{i}$ : Trabajo en proceso de la compañía $i$ en $t$,
$Y_{i}^{i}$ inventario de partes/productos terminados en el búfer de la compañía $i$, en $t$,
$S_{i}^{i}$ : tasa de envíos de la compañía $i$ a la compañía $i+1$, en el período $t$,
$O_{i}^{i}$ : unidades salientes del inventario de trabajo en proceso de la compañía $i$ en el período $t$, o sea, tasa de terminación de unidades,
$I_{t}^{i}$ : unidades entrantes al proceso de la compañía i, en el período t, o sea, tasa de aprovisionamiento (producción) de la compañía i,

v Variables del flujo de información: 
D. :Pedidos de clientes finales a la CS (en la compañía final) en el período $t$,

$B$ : :pedidos de clientes finales pendientes de satisfacer en el período $t$,

$D O$ : cantidad de órdenes finalmente enviadas a los clientes finales (desde la compañía final) a los clientes finales en el período $t$,

$A P C_{t}$ :número de tarjetas de producción disponibles ent,

$O P_{t}$ :órdenes de aprovisionamiento lanzadas (por la primera compañía) en el período $t$,

$P B, \quad$ :lista de pedidos que debieron, pero no fueron lanzados a producción a tiempo ent,

$D S$ : entregas deseadas a los clientes finales en el período $t$,

DPO: ordenes de producción deseadas a circular en la CS en el período $t$,

$T Y^{i}$ : partes/productos finales totales disponibles en la compañía $i$, ent,

\section{■ Parámetros del modelo:}

$L^{i} \quad$ :tiempo de ciclo para que una unidad en el inventario de trabajo en proceso se acumule en el inventario de partes/productos finales de la compañía i,

$M L P^{i}$ :máxima carga de partes a ser procesadas en la compañíai,

$\alpha \quad$ :factor de suavización de la demanda,

$U C^{i}$ : unidades por contenedor en la compañía i (se asume el mismo valor UC para todas las compañías),

$T N P C^{\prime}$ :número total de tarjetas de producción a circular en el sistema.

SS :tiempo deseado de permanencia de una unidad de material, como inventario a mano en un nodo cualquiera (se asume la misma política para todos),

$\beta_{s} \quad$ :coeficiente de ajuste fraccional del inventario a mano,

$\beta_{s L} \quad$ :Coeficiente de ajuste fraccional del inventario de trabajo en proceso.
Para comparar el rendimiento de las dos alternativas de GCS, en primer lugar se retoman las premisas del modelo de la CS TI de acuerdo a Rubiano (2003), en el cual se asume que (a) los socios de la cadena toman decisiones de ordenar individuales, lo cual implica un control descentralizado en la cadena, (b) las ordenes en la cadena de suministro son visibles en tiempo real, y procesadas en concordancia con ello, y (c) los socios de la cadena ganan acceso a información adicional que ellos no controlan, y la usan en su proceso del planeación (por ejemplo en pronósticos de las compañías finales, los inventarios a mano y los inventarios de trabajo en proceso de los otros miembros de la cadena, los pedidos pendientes, etc.). En segundo lugar se presentan las ecuaciones del modelo de la CS COMWIP y se comparan con las ecuaciones del modelo de la CS TI, indicando aquellas que son diferentes a su homóloga respectiva en Rubiano (2003). Seguidamente, se valida el modelo de la CS COMWIP, y finalmente se simulan y se comparan ambos sistemas para una serie de escenarios diseñados.

En este estudio se asegura que para cada escenario particular, ambos sistemas operan en sus mejores condiciones posibles (es decir con el mejor valor posible de sus parámetros, cuyos criterios de medición se presentan en la sección 6). Esto se hará mediante una optimización multiparamétrica para cada caso particular.

En la Figura 3, se puede apreciar claramente la



Figura 3. Diagrama de influencia básico de la CS COMWIP para las variables de la compañía [i] y la compañía [n] 
relación entre las principales variables del flujo de información de la CS COMWIP (líneas delgadas) y las variables del flujo materiales (líneas gruesas).

Seguidamente se resumen las ecuaciones para el modelo de la CS COMWIP, para una compañía $i$ en el período $t$.

Pedidos recibidos: toma el valor generado por la demanda final:

$$
D_{t}=O P_{t}^{\text {Clientes }}
$$

Entregas deseadas:

Equivalentes a los pedidos recibidos:

$$
D S_{t}=D_{t}
$$

Órdenes deseadas a lanzar a la primera compañía proveedora: $D P O_{t}=D_{t}$

Entregas:

$S_{t}^{i}= \begin{cases}M I N\left(T Y_{t}^{i}, M L P^{i+1} / L^{i+1}\right) & \text { Para las compañías } \\ i=1, \ldots, n-1 \\ M I N\left(T Y_{t}^{n}, D S_{t}\right) & \text { Para la compañía } \\ i=n\end{cases}$

Inventario de producto

Terminado total disponible: $\quad T Y_{t}^{i}=Y_{t}^{i}+O_{i}$

El Inventario a mano:

$$
Y_{t}^{i}=Y_{t-1}^{i}+O_{t}^{i}-S_{t}^{i}
$$

Tasa de terminación del IPP: $\quad O_{t}^{i}=I_{t \cdot L^{i}}^{i}$

Tasa de aprovisionamiento /producción: es la misma cantidad de órdenes enviadas por la compañía $i-1$ :

$$
I_{t}^{i}=S_{t}^{i-1}
$$

Abastecimiento a la primera compañía: simplemente se hace equivalente a las órdenes colocadas por parte de ella misma:

$$
I_{t}^{i}=O P_{t}
$$

Inventario de trabajo en proceso: $P_{t}^{i}=P_{t-1}^{i}+I_{t}^{i}{ }^{-} O_{t}^{i}$ como ventas perdidas, se

Originan sólo en la compañía

final de la cadena: $\quad B_{t}=B_{t \cdot l}+D_{t}-D O_{t}$

$D O$, es la información del suministro a los clientes finales:

$$
D O_{t}=S_{t}^{n}
$$

Cuando la disponibilidad de tarjetas limita el lanzamiento de órdenes a la CS, se acumula un registro de órdenes no lanzadas a tiempo a la primera compañía, $P B_{t}$ :

$$
P B_{t}=P B_{t-t}+D_{t}-O P_{t}
$$

Órdenes de aprovisionamiento (decisión fundamental), es el mínimo entre las órdenes de producción deseadas $D P O_{t}$, las tarjetas disponibles convertidas en unidades, asumiendo la misma cantidad de unidades por contenedor (UC) en todas las compañías, $A P C_{t}^{*} U C$, y la máxima tasa de producción de la primera compañía $M L P^{1} / L^{1}$ :

$O P_{t}=M A X\left(M I N\left(A P C_{t} * U C, D P O_{t}, M L P^{\prime} / L^{t}\right), O\right)(13)$

Tarjetas de producción

disponibles:

$$
A P C_{t}=T N P C-\left(\sum_{i=1}^{n} P_{t}^{i}+\sum_{i=1}^{n} Y_{t}^{i}\right) / U C
$$

Otra decisión fundamental de la cadena de suministro COMWIP, la constituye la estimación del número total de tarjetas a circular en la CS, TMPC. Este valor se asume como un parámetro de búsqueda, y se calcula estableciendo una política de optimización en la cual se maximizan las entregas a los clientes finales (throughput), mientras se minimizan los inventarios globales.

Órdenes no satisfechas a

tiempo: son consideradas 


\section{VALIDACIÓN DE LOS PATRONES DE COMPORTAMIENTO PARA LAS PRINCIPALES VARIABLES DE LA CS CONWIP}

Para validar el comportamiento de las principales variables del modelo de la estrategia COMWIP en la CS, se utilizó un ejemplo similar al usado por Sterman (1984) en el juego de la cerveza, esta vez con demanda variable. Son 4 compañías en serie (Figura 3): un proveedor, un primer fabricante, un segundo fabricante, y los canales de distribución y detal. Los resultados de los patrones de comportamiento son presentados principalmente para estudiar el impacto del control centralizado y el inventario total limitado en medidas de rendimiento globales, mientras se trata la CS como una sola célula.

La demanda final se genera como un número de una distribución normal, con media asumida de 4 unidades/semana y desviación estándar $(D E)$ de 4 unidades. El valor de las unidades por contenedor $(U C)$ es 1 , y el factor de suavización $(\alpha)$ es 0.2. El número total de tarjetas de producción es 68. Las condiciones iniciales para los inventarios en las compañías son:

IPP: $P_{\circ}^{i}=8$ cajas de cerveza, para $i=1$ a $n$,

IPT: $Y_{\circ}^{i}=12$ cajas de cerveza, para $i=1 \mathrm{a} n$.

Los valores adoptados para los demás parámetros operativos se incluyen en la figura 4.



Figura 4. Ejemplo de la CS seleccionada para la validación del modelo

La Figura 5 muestra el comportamiento de las órdenes lanzadas por parte del fabricante y las entregas a los cliente finales (Throughput). En el escenario escogido para la validación, la restricción de carga máxima no limita la política de lanzamiento de órdenes, sin embargo las tarjetas de producción disponibles (figura 5) podrían hacerlo (como ocurre en las semanas $8^{a}$ y $11^{a}$ ). De tomas maneras, la política de lanzamiento de órdenes permite lanzar más que suficiente cantidad de materias primas al proveedor, de tal manera que ellas fluyen y llegan a tiempo al búfer de productos terminados de los canales de distribución, para atender la demanda.

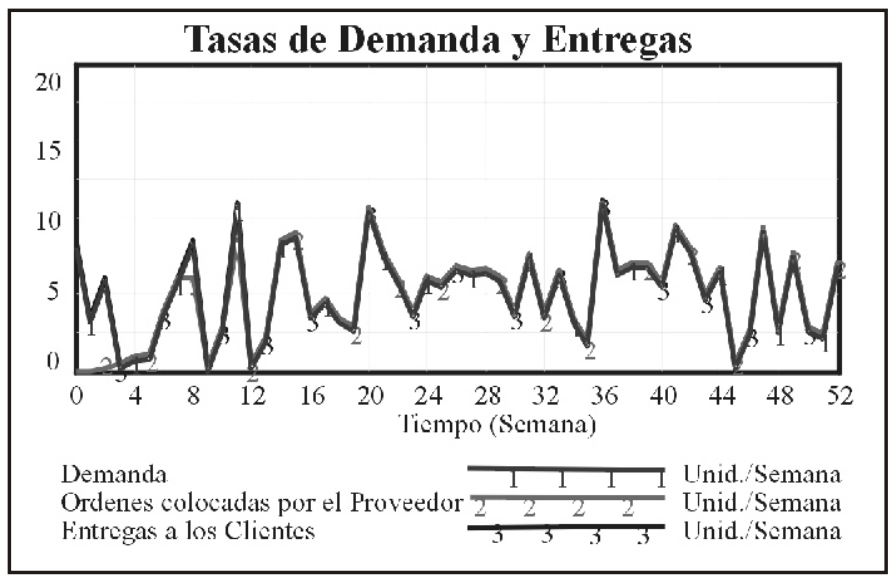

Figura 5. Tasas de demanda y entregas

La 6 junto con la figura 5 muestran que: (a) Durante las primeras tres semanas no es necesario lanzar órdenes debido a las cantidades de inventarios iniciales establecidas; (b) se cumple la condición $T N P C=A P C_{t}+\left(P_{t}^{i}+Y_{t}^{i}\right) / U C$; y $(c)$ a partir del momento en que los inventarios totales en la cadena alcanzan el estado de equilibrio ocurre que, a medida que llegan nuevos

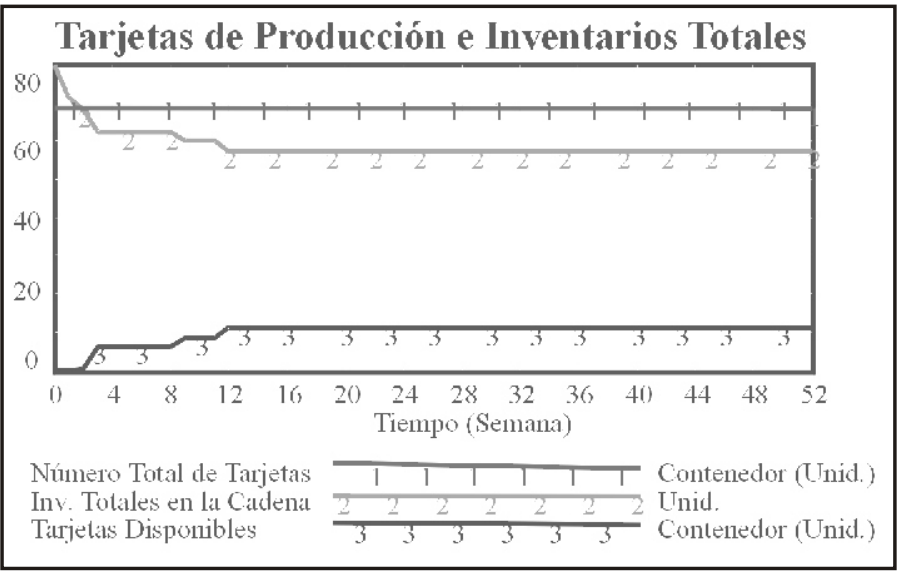

Figura 6. Tarjetas de Producción e Inventarios Totales 
pedidos, un número suficiente de tarjetas siempre está disponible para lanzar las órdenes necesarias, producir y atender toda la demanda.

De acuerdo con estos resultados obtenidos con el modelo de la CS CONWIP, se puede afirmar que se han logrado reproducir las principales características de una estrategia de control COMWIP (Spearman et al. 1990) en un modelo de CS administrada centralmente (de acuerdo con Spearman et al. 1990).

\section{SIMULACIÓN Y COMPARACIÓN DE LAS ESTRATEGIAS DE GCS}

En esta sección se presentan una serie de resultados para comparar las estrategias CS COMWIP y CS TI con respecto a una serie de medidas de rendimiento operativos y financieros. Los resultados se presentan a nivel global de la CS para ambas estrategias. La CS consiste en cuatro compañías (Fabricante, Distribuidor, Mayorista y Detallista) tal como los plantea Sterman (1984) en el artículo del juego de la cerveza. Las simulaciones corren durante 52 semanas.

Para las cuatro situaciones consideradas en el estudio, en la tabla 1 se presentan los valores de los parámetros de la demanda, así como los valores iniciales para los inventarios de trabajo en proceso y finales en todas las compañías.

Tabla 1. Parámetros especificados para la demanda final y condiciones iniciales de los inventarios

\begin{tabular}{|c|c|c|c|c|c|c|}
\hline \multicolumn{6}{|c|}{$\begin{array}{c}\text { Demanda (unidades / semana) } \\
\text { (Parámetros de distribución normal asumida) }\end{array}$} & \multicolumn{2}{c|}{$\begin{array}{c}\text { Condiciones } \\
\text { iniciales } \\
\text { (Unidades) }\end{array}$} \\
\hline Situación & Mínimo & Máximo & Media & $\begin{array}{c}\text { Desviación } \\
\text { estándar } \\
\text { (DE) }\end{array}$ & IPP & IPT \\
\hline 1 & 0 & 8 & 4 & 4 & 8 & 12 \\
2 & 0 & 8 & 4 & 3 & 8 & 12 \\
3 & 0 & 8 & 4 & 2 & 4 & 8 \\
4 & 0 & 8 & 4 & 1 & 3 & 7 \\
\hline
\end{tabular}

1 La validación del modelo de la CS TI se realizó en Rubiano (2003)
Los escenarios o situaciones para las simulaciones se construyen variando para cada valor de la DE de la demanda, la carga máxima de partes MLP (desde 30 hasta 10 unidades para todas las compañías). Una vez realizadas las simulaciones, se presentan los resultados de las medidas de rendimiento seleccionadas para la posterior comparación de las dos estrategias.

Para obtener los valores de los parámetros para cada estrategia, produciendo su mejor rendimiento en cada escenario, se usó una técnica de optimización numérica de búsqueda directa, la cual no necesita evaluar el gradiente y es muy apropiada para el análisis de la dinámica de sistemas de control no lineales complejos. Esta técnica es el método de Powell modificado (Powell 1968), el cual es bien conocido entre los métodos de búsqueda directa ${ }^{2}$ por su uso para obtener una convergencia final rápida.

La tabla 2 muestra las variables equivalentes a optimizar y los parámetros de búsqueda para cada optimización de las estrategias, también muestra las restricciones del sistema, iguales para ambos casos. Se asignaron pesos para equilibrar los órdenes de cifras numéricas de las variables a optimizar, esto con el criterio de asegurar las mismas altas tasas de cobertura de la demanda (nivel de servicio mediante el cumplimento de las entregas) en ambas estrategias en todos los escenarios.

El conjunto de gráficos siguiente, muestra los resultados comparativos globales con respecto a las medidas de rendimiento operativo enfatizando en los resultados de las compañías

\footnotetext{
2 La idea básica que respalda al método Powell (Powell, 1964) es descomponer la minimización M-dimensional en $M$ problemas de minimización uni-dimensionales. Luego, para cada problema unidimensional se lleva a cabo una búsqueda binaria para encontrar el mínimo local dentro de un rango dado. Además, en las iteraciones subsiquientes se realiza una estimación de las mejores direcciones a usar para las búsquedas uni-dimensionales. Sin embargo, para algunos problemas, no siempre se aseguran soluciones óptimas, debido a que los vectores de dirección no siempre son linealmente independientes. Para superar esto, el método fue revisado (Powell 1968) introduciendo nuevos criterios para la formación de vectores de dirección linealmente independientes; este método revisado es llamado "El Método de Powell Modificado".
} 
iniciales, que es donde más impacta fundamentalmente la problemática de la variabilidad en la CS.

Según los resultados presentados en la figura 7 , a medida que la carga máxima permitida de partes al fabricante decrece, también la cantidad media de órdenes lanzadas por el fabricante disminuye en ambas estrategias. Una vez más, esto es debido a los límites impuestos por la máxima capacidad de producción sobre las respectivas políticas de lanzamiento de órdenes. Sin embargo a medida que la DE de la demanda disminuye, el comportamiento de las órdenes lanzadas es análogo al del número total de tarjetas de producción y el stock de seguridad.

Por otro lado, los resultados indican que en todos los escenarios la CS COMWIP necesita lanzar menores cantidades medias de órdenes que la CS TI, cuando ambas estrategias ofrecen similares niveles de servicio, pero esta diferencia es mayor en escenarios con cargas máximas y $D E$ de la demanda relativamente bajas. Este resultado se debe a que la CS COMWIP ordena cantidades exclusivamente para satisfacer la previsión de la demanda y la CS TI intenta pedir para mantener un inventario meta (mayor que la previsión de la demanda), el cual es un aspecto importante de este resultado.

Tabla 2. Estrategias de GCS y criterios de optimización

\begin{tabular}{|c|c|c|c|}
\hline & \multicolumn{3}{|c|}{ Estrategia de GCS } \\
\hline & $\begin{array}{l}\text { Totalmente integrada } \\
\text { (FI CS) }\end{array}$ & CS COMWIP & $\begin{array}{l}\text { Criterios de } \\
\text { Optimización }\end{array}$ \\
\hline \multirow{3}{*}{$\begin{array}{l}\text { Variables a } \\
\text { Optimizar } \\
\text { (Función de } \\
\text { Optimización) }\end{array}$} & - Entregas (Throughput) & - Entregas & - Maximizar \\
\hline & $\begin{array}{l}\text { - Órdenes deseadas } \\
\text { de Producción Totales }\end{array}$ & $\begin{array}{l}\text { - Tarjetas de } \\
\text { Producción } \\
\text { Disponibles }\end{array}$ & - Minimizar \\
\hline & - Inventarios Totales & $\begin{array}{l}\text { - Inventarios } \\
\text { Totales }\end{array}$ & - Minimizar \\
\hline $\begin{array}{l}\text { Parámetros de } \\
\text { Búsqueda }\end{array}$ & $S S, \beta_{\mathrm{s}}, \beta_{\mathrm{sL}}$ & $T N P C$ & \\
\hline Restricciones & Máxima carga (MLP) & Máxima carga (MLP) & \\
\hline
\end{tabular}

Tabla 3. Valores de los parámetros constantes en todas las simulaciones

\begin{tabular}{|l|c|c|c|c|c|}
\hline \multicolumn{1}{|c|}{ Parámetros } & Proveedor & $\begin{array}{c}\text { 1er. } \\
\text { Fabricante }\end{array}$ & $\begin{array}{c}\text { 2do. } \\
\text { Fabricante }\end{array}$ & $\begin{array}{c}\text { Canales de } \\
\text { Distribución }\end{array}$ & Unidades \\
\hline - Operativos & & & & & \\
\hline Tiempo de Ciclo, $L^{i}$ & 2 & 2 & 2 & 2 & Semana \\
\hline Unidad por Contenedor, $U C^{i}$ & 1 & 1 & 1 & 1 & Unidad \\
\hline Factor de suavización, $\alpha^{I}$ & 0.2 & 0.2 & 0.2 & 0.2 & Adimens. \\
\hline - Financieros & & & & & \\
\hline Precio por unidad, $P m_{t}^{i}$ & 120 & 208 & 303 & 383 & \$/Unidad \\
\hline Período de cobro, $d s o(i)$ & 2 & 2 & 2 & 2 & Semana \\
\hline Margen de utilidad, $m r_{t}^{i}$ & 0.5 & 0.4 & 0.3 & 0.2 & Adimens. \\
\hline
\end{tabular}




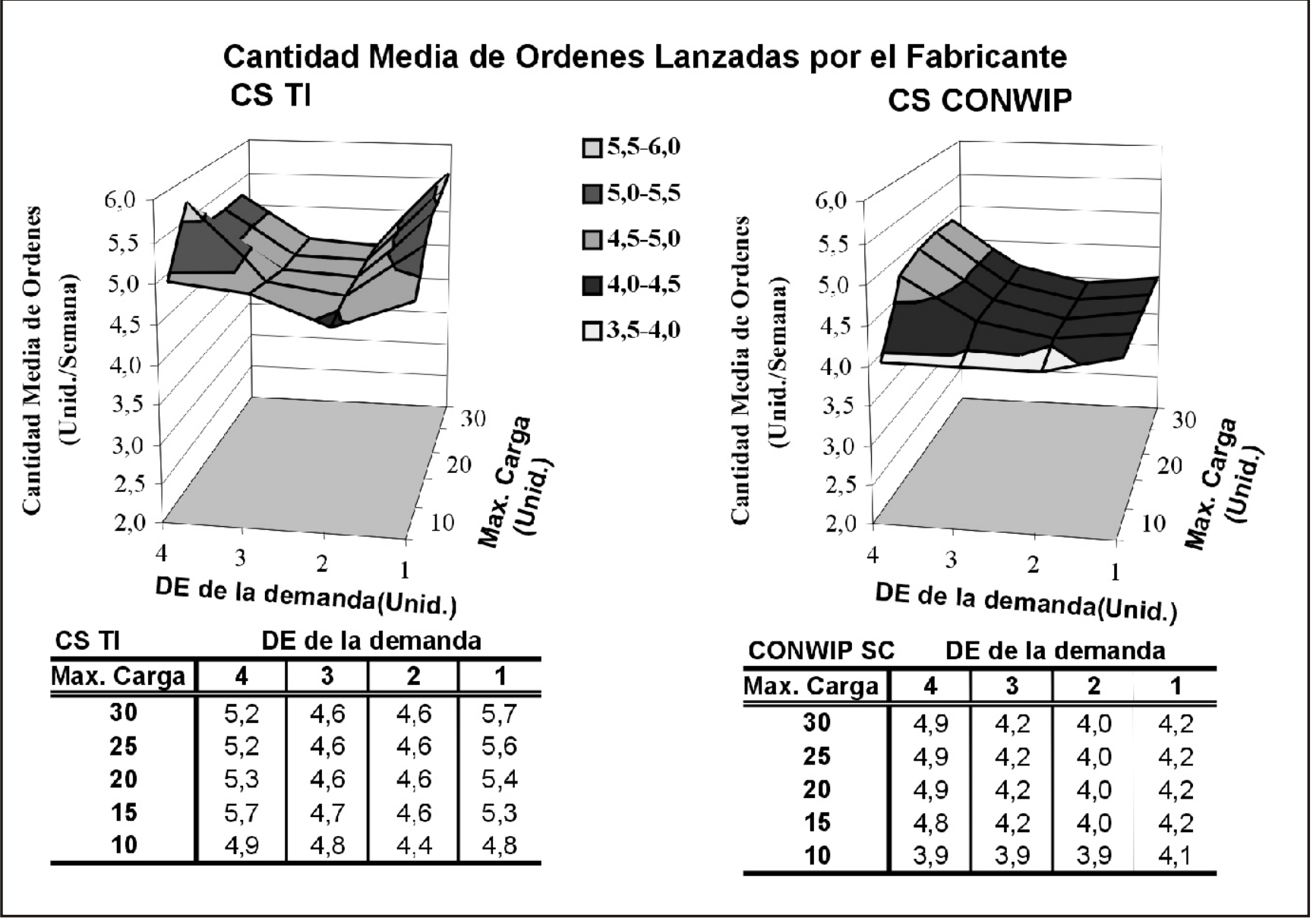

Figura 7. Resultados para la Cantidad Media de Órdenes Lanzadas por el Fabricante

La figura 8 ilustra que, lo mismo que para las cantidades pedidas, a medida que la carga máxima permitida de partes al fabricante disminuye, la desviación estándar de las órdenes lanzadas por el fabricante también disminuye en ambas estrategias. En general la CS COMWIP sufre menores impactos por parte de la variabilidad de la demanda.

El posible efecto "bullwhip" que pudiera generarse en la CS COMWIP, se mitiga en gran medida, principalmente por:

(a) La gestión centralizada de la previsión de la demanda y los inventarios, lo cual evita en parte que una compañía dependa de otra, y

(b) la información respectiva es enviada desde las compañías directamente al control central.
La figura 9 muestra una de las diferencias más significativas entre las dos estrategias. Mediante la CS COMWIP se logran menores niveles globales de inventario medio en todos los escenarios. Este resultado evidencia una ventaja esperada de la estrategia COMWIP sobre la TI, esto es, mayor eficiencia de los inventarios al ofrecer un nivel de servicio similar.

Finalmente, con respecto a los costos de administración de los inventarios (costos de mantener + costos de penalización de las roturas), la estrategia COMWIP es más robusta al incurrir en menos costos en todas las situaciones (figura 10). Estos resultados demuestran un flujo de materiales más uniforme, control de inventarios más fácil y mejor sincronización entre las compañías. 


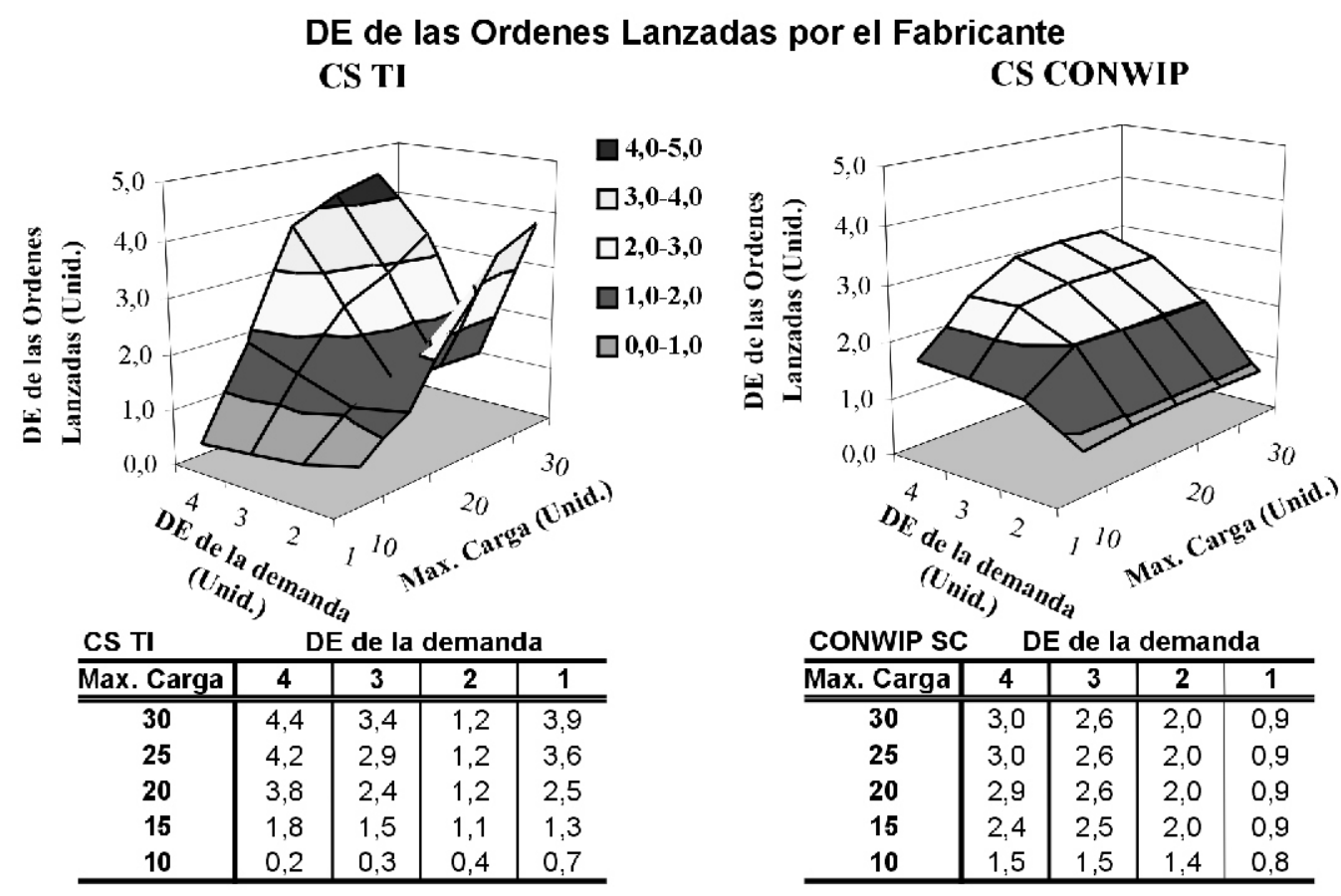

Figura 8. Resultados para la DE de las Órdenes Lanzadas por el Fabricante

Cantidad media de Trabajo en Proceso + Inv. de Prod. Terminados en la Cadena

CS TI

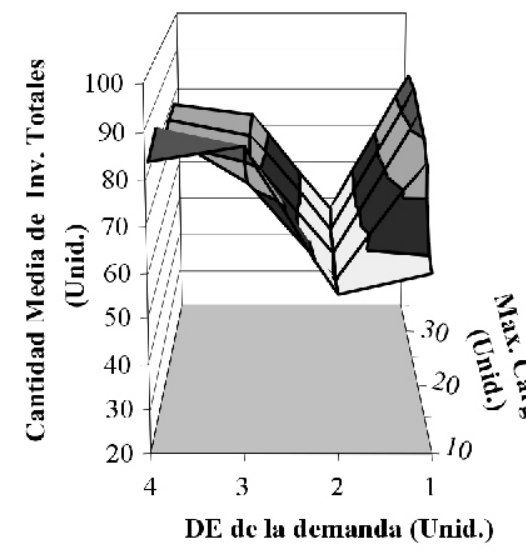

\begin{tabular}{c|c|c|c|c} 
CS TI & \multicolumn{4}{c}{ DE de la demanda } \\
\hline Max. Carga & $\mathbf{4}$ & $\mathbf{3}$ & $\mathbf{2}$ & $\mathbf{1}$ \\
\hline \hline $\mathbf{3 0}$ & 77 & 75 & 50 & 85 \\
$\mathbf{2 5}$ & 78 & 74 & 50 & 85 \\
$\mathbf{2 0}$ & 77 & 73 & 50 & 84 \\
$\mathbf{1 5}$ & 84 & 72 & 50 & 76 \\
$\mathbf{1 0}$ & 82 & 85 & 52 & 57 \\
\hline
\end{tabular}

\section{CS CONWIP}

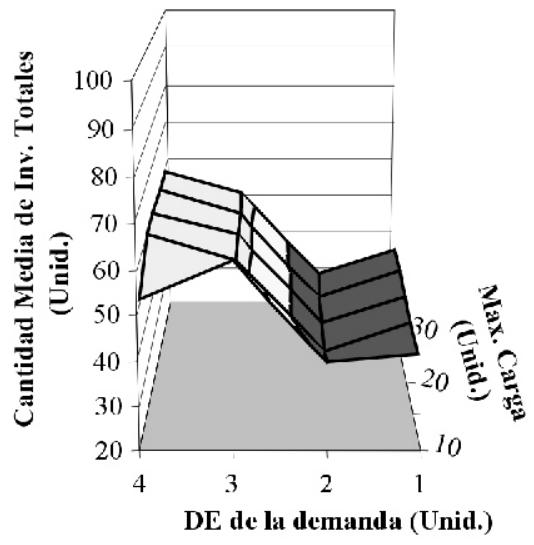

CONWIP SC DE de la demanda

\begin{tabular}{c|c|c|c|c}
\hline Max. Carga & $\mathbf{4}$ & $\mathbf{3}$ & $\mathbf{2}$ & $\mathbf{1}$ \\
\hline \hline $\mathbf{3 0}$ & 59 & 53 & 31 & 38 \\
$\mathbf{2 5}$ & 59 & 53 & 32 & 38 \\
$\mathbf{2 0}$ & 59 & 53 & 31 & 38 \\
$\mathbf{1 5}$ & 60 & 53 & 31 & 38 \\
$\mathbf{1 0}$ & 51 & 59 & 36 & 38 \\
\hline
\end{tabular}

Figura 9. Resultados para el Inventario Total Medio 
Finalmente, con respecto a los costos de administración de los inventarios (costos de mantener + costos de penalización de las roturas), la estrategia COMWIP es más robusta al incurrir en menos costos en todas las situaciones
(Figura 10). Estos resultados demuestran un flujo de materiales más uniforme, control de inventarios más fácil y mejor sincronización entre las compañías.

\section{Costo medio en Inventarios en la Cadena}

CS TI

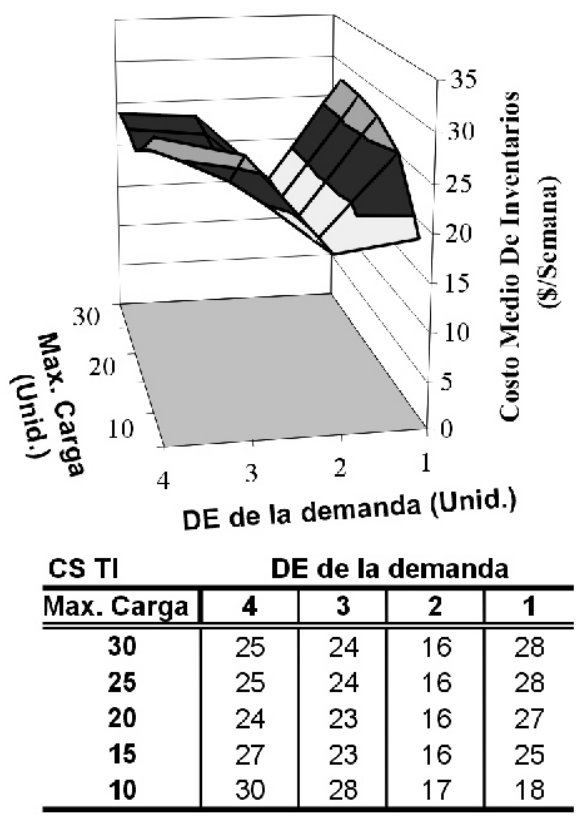

CS CONWIP
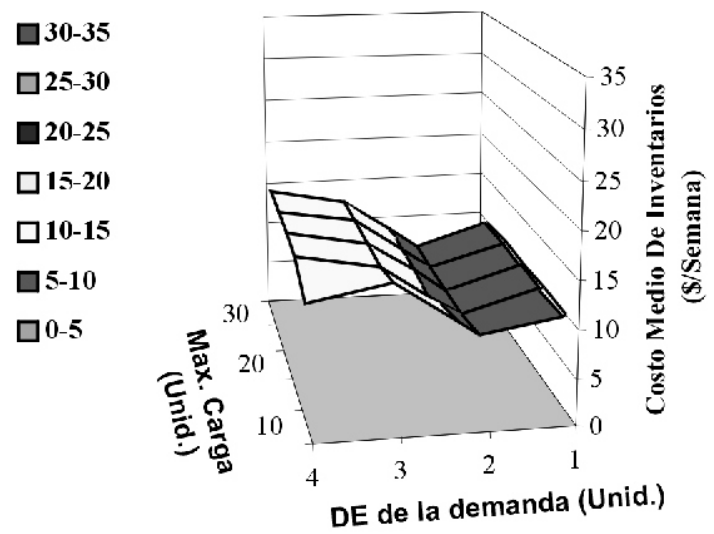

CONWIP SC DE de la demanda

\begin{tabular}{c|c|c|c|c}
\hline Max. Carga & $\mathbf{4}$ & $\mathbf{3}$ & $\mathbf{2}$ & $\mathbf{1}$ \\
\hline \hline $\mathbf{3 0}$ & 15 & 13 & 8 & 10 \\
$\mathbf{2 5}$ & 15 & 13 & 8 & 10 \\
$\mathbf{2 0}$ & 15 & 13 & 8 & 10 \\
$\mathbf{1 5}$ & 15 & 13 & 8 & 10 \\
$\mathbf{1 0}$ & 13 & 14 & 9 & 10 \\
\hline
\end{tabular}

Figura 10. Resultados para los Costos Medios de Inventarios en la Cadena

\section{CONCLUSIONES}

En este artículo se evaluó el rendimiento de la estrategia de CS centralizada mediante el sistema de gestión COMWIP en un ambiente variable, y se comparó este rendimiento al de la CS totalmente integrada (descentralizada). Con base en los resultados obtenidos, parece ser que la CS gestionada mediante la estrategia COMWIP representa un control centralizado a lo largo de la cadena que puede ofrecer ventajas en el rendimiento, en comparación con la CS TI descentralizada. Una CS COMWIP ofrece las siguientes ventajas sobre la CS TI, cuando ellas ofrecen niveles de servicio similares:

1. Control más fácil del inventario de producto en proceso, puesto que los flujos de materiales y partes están controlados y limitados centralmente.

2. La CS COMWIP necesita hacer menor cantidad media de pedidos que la CS TI especialmente en escenarios con relativa carga máxima baja y baja desviación estándar de la demanda.

3. Generalmente la política de ordenar en la CS TI es más vulnerable a medida que aparece variabilidad en la demanda y la capacidad de carga en las compañías no es muy pequeña. 
En CS COMWIP, la gestión centralizada de la demanda e inventarios mitiga la amplificación de la variabilidad de la demanda, contribuyendo a que las cantidades pedidas se ajusten a las necesidades reales de flujo de material.

4. La estrategia CS COMWIP ofrece niveles medios de inventarios totales, potencialmente menores (mayor eficiencia) propiciando que la cadena del suministro maneje menores costos medios de inventarios.

En resumen, aunque se puede encontrar un poco de resistencia al cambio por parte de las empresas que se integran en una CS, la exploración del uso del sistema CONWIP para la GCS, podría ser una fuente de beneficios potenciales en un futuro cercano.

\section{BIBLIOGRAFÍA}

1. HOPP, W. J. and Spearman, M. L. 1996. Factory Physics. (Chicago, USA: Irwin).

2. MUCKSTADT, J. A. and Tayur, S. R. 1995a. A comparison of alternative kanban control mechanisms: I, Background and structural results. IIE Transactions. 27(1). 140-150.

3. MUCKSTADT, J. A. and Tayur, S. R. 1995b. A comparison of alternative kanban control mechanisms: II, Experimental results. IIE Transactions. 27(1). 151-161.

4. MUCKSTADT, J. A. and Tayur, S. R. 1995a. A comparison of alternative kanban control mechanisms: I, Background and structural results. IIE Transactions. 27(1). 140-150.

5. POWELL, M. J. D. 1964. An Efficient Method for Finding the Minimum of a Function of Several Variables Without Calculating Derivatives. Computer Journal. 7 (2). 155-62.
6. POWELL, M. J. D. 1968. On the calculation of orthogonal vectors. Computer Journal. 11(2). 302-304.

7. RODERICK, L. M.; Phillips, D. T. and Hogg, G. L. 1990. A comparison of order release strategies in production control systems. International Journal of Production Research. Submitted for publication.

8. RUBIAMO, O. 2003. Mejora del Rendimiento Operativo y Financiero de las Cadenas de Suministro mediante el uso de las Herramientas de Colaboración basadas en Internet. Un enfoque Sistémico. Ingeniería y Competitividad. 4(2). 34-41. Facultad de Ingeniería, Universidad del Valle, Cali, Colombia.

9. SPEARMAM, M. L.; Woodruff, D. L. and Hopp, W. J. 1990. COMWIP: a pull alternative to kanban. International Journal of Production Research. 28. 879-894.

10. SPEARMAM, M. L. and Zazanis M. A. 1992. Push and Pull Production systems: issue and comparison. Operations Research. 40(3). 521-532.

11. SPEARMAM, M. L. and Zazanis M. A. 1992. Push and Pull Production systems: issue and comparison. Operations Research. 40(3). 521-532.

12. STERMAM, J. 1984. Instructions for Running the Beer distribution Game (D-3679). Sloan School of Management, MIT. 